\title{
Gender differences in honesty: Groups versus individuals
}

\author{
Gerd Muehlheusser ${ }^{\mathrm{a}, \mathrm{b}, \mathrm{c}}$, Andreas Roider ${ }^{\mathrm{b}, \mathrm{c}, \mathrm{d}, \mathrm{e}, *}$, Niklas Wallmeier $^{\mathrm{a}}$ \\ a Department of Economics, University of Hamburg, Von-Melle-Park 5, 20146 Hamburg, Germany \\ ${ }^{\mathrm{b}}$ IZA, Germany \\ ${ }^{\mathrm{c}}$ CESifo, Germany \\ d Department of Economics, University of Regensburg, Universitätsstr. 31, 93040 Regensburg, Germany \\ ${ }^{\text {e } C E P R, U K}$
}

\section{H I G H L I G H T S}

- We analyze gender effects in the lying behavior of groups and individuals.

- We extend the die-rolling experiment of Fischbacher and Föllmi-Heusi (2013).

- There are no pronounced gender effects under individual decision-making.

- Strong gender effects emerge under joint decision-making in groups.

- There is more lying in male groups and mixed groups than in female groups.

\section{A R T I C L E I N F O}

\section{Article history:}

Received 19 September 2014

Received in revised form

4 December 2014

Accepted 12 December 2014

Available online 10 January 2015

\section{JEL classification:}

C91

C92

J16

\section{Keywords:}

Unethical behavior

Lying

Group decisions

Gender effects

Experiment

\begin{abstract}
A B S T R A C T
Extending the die rolling experiment of Fischbacher and Föllmi-Heusi (2013), we compare gender effects with respect to unethical behavior by individuals and by two-person groups. In contrast to individual decisions, gender matters strongly under group decisions. We find more lying in male groups and mixed groups than in female groups.
\end{abstract}

(c) 2015 Elsevier B.V. All rights reserved.

\section{Introduction}

Unethical behavior is a ubiquitous feature in many economic contexts, and a number of recent experimental studies have analyzed lying as one prominent type of unethical behavior. For example, in Fischbacher and Föllmi-Heusi (2013) individuals are

\footnotetext{
* Corresponding author at: Department of Economics, University of Regensburg, Universitätsstr. 31, 93040 Regensburg, Germany. Tel.: +499419432701.

E-mail addresses: gerd.muehlheusser@wiso.uni-hamburg.de

(G. Muehlheusser), andreas.roider@ur.de (A. Roider),

niklas.wallmeier@wiso.uni-hamburg.de (N. Wallmeier).
}

asked to report the (privately observed) realization of a die roll that determines their payoff. Evidence for lying (on the aggregate level) is then obtained by comparing the actual payoff distribution with the uniform distribution, which would result under truth-telling. Other studies have analyzed lying using the sender-receiver setup of Gneezy (2005). All in all, there is strong evidence for lying, but often not to the maximal extent possible; suggesting that there are private costs associated with such unethical behavior (Gneezy, 2005; Charness and Dufwenberg, 2006; Erat and Gneezy, 2012; Gibson et al., 2013).

With respect to gender differences, it seems that males are somewhat more prone to lying than females, but often the effect is small or not statistically significant (Dreber and Johannesson, 
2008; Childs, 2012; Erat and Gneezy, 2012; Houser et al., 2012; Conrads et al., 2013, 2014; Abeler et al., 2014). ${ }^{1}$

So far, the literature on lying behavior has mainly analyzed decisions by individuals; possibly in strategic interaction with other individuals as in tournaments (see e.g., Conrads et al., 2014). However, in many settings, a group of individuals must reach a decision jointly, e.g., decision-making by committees in economic, social, or political organizations. In fact, there is growing evidence from contexts other than lying that groups often decide markedly different than individuals (for surveys, see Charness and Sutter, 2012; Kugler et al., 2012). On the one hand, groups are better at solving cognitive tasks and act more selfishly (see e.g., Maciejovsky et al., 2013; Bornstein et al., 2004; Falk and Szech, 2013). That suggests that groups might be more willing to realize the potential monetary gains from lying. On the other hand, there is evidence that "moral reminders" reduce dishonesty (Pruckner and Sausgruber, 2013). Hence, discussions within groups might lead them to lie less. Taken together, it seems a priori unclear whether lying is more prevalent in groups compared to individuals. Moreover, for the lying behavior of groups their gender composition might matter (see e.g., Dufwenberg and Muren, 2006, where gender composition affects groups' giving in a dictator game). Consequently, this paper aims at providing insights on the unethical behavior of groups and individuals, and the role of gender in this context. Gender composition is found to be particularly important under group decisionmaking. In our view, this has interesting implications for the design of decision-making (and monitoring) processes in organizations.

\section{Experimental design}

We extend the simple and widely used die rolling experiment of Fischbacher and Föllmi-Heusi (2013), where subjects decide autonomously and anonymously about their (lying) behavior, to a setting where decisions are made jointly in groups. We consider a treatment $G$ where randomly formed groups of two subjects need to coordinate on both who rolls the die and on which realization to declare. As a control treatment $I$, we replicate the setup of decision-making by individuals as in Fischbacher and Föllmi-Heusi (2013). Subjects were randomly assigned to treatments (and in treatment $G$, to groups).

The experiment was conducted at the University of Regensburg in June 2014. Participants were recruited through an introductory undergraduate course in economics (economics majors and minors and business majors). ${ }^{2}$ Subjects were first asked to complete an unrelated questionnaire inside the lecture hall. They were instructed (i) that their payoff for filling out the questionnaire would be either $0,1,2,3,4$, or 5 Euros, and (ii) that the exact amount would be determined in a second phase of the experiment outside the lecture hall, where they would receive further instructions. We made it clear that payoffs would be completely independent from their answers in the questionnaire, and that their behavior in the experiment would remain anonymous.

The die rolling experiment was then played in paper-pencil style in fifteen booths outside the lecture hall that ensured complete privacy of decision-making. Subjects waited inside the lecture hall at their seats, and were only allowed to proceed outside when booths became vacant. Inside the booth, subjects found a fair, six-sided die, a pen, instructions, an anonymous answer sheet (on which the realization of the die roll was to be declared), and a receipt form for each subject. Translations of the instructions and the

\footnotetext{
1 For surveys on gender differences in a variety of economic contexts, see e.g., Eckel and Grossman (2008) and Croson and Gneezy (2009).

2 As a show-up fee, students who agreed to participate (which all did) received a small bonus towards their final exam.
}

answer sheet are included in the Supplementary Material. As each booth contained one die and one answer sheet only, in treatment $G$, subjects had to make a joint declaration, and they were aware that each of them would receive the declared payoff. ${ }^{3}$ Afterwards, subjects proceeded to the cashier desk. They handed in the anonymous questionnaire(s) and the anonymous answer sheet, where it was checked that the declared amounts coincided with those on the receipt form(s). Then each subject went to privately collect his/her payment. As in Fischbacher and Föllmi-Heusi (2013), subject $i$ 's payment (in Euros) $\pi_{i}$, is related to the declared outcome of the die roll $r \in\{1, \ldots, 6\}$ as follows: $\pi_{i}=r$ for all $r \leq 5$ and $\pi_{i}=0$ for $r=6$. In total, there were 228 participants ( 124 female, 104 male) of which 108 (120) participated in treatment $I(G)$. The whole experiment took about 2 hours.

\section{Results}

Table 1 summarizes the distribution of payoffs in the two treatments. In line with the previous literature, a sizeable amount of lying also occurs in our setting. First, the average payoffs in treatments $G$ and $I$ are 3.47 and 3.48, respectively. Hence, they virtually take the same value (3.51) as in the baseline (individualistic) treatment of Fischbacher and Föllmi-Heusi (2013). Both payoff distributions differ significantly from the uniform distribution that would result under truthful reporting leading to an average payoff of 2.50 ( $p<0.001$, two-sided one-sample Kolmogorov-Smirnov (KS) tests). These results are driven mainly by the high frequency of reported 4's and 5's. Comparing our two treatments reveals that - when considering all observations - their payoff distributions do not differ significantly at conventional levels according to a twosided Mann-Whitney U (MWU) test. ${ }^{4}$ However, as shown next, this result masks substantial gender differences. As displayed in Fig. 1(a), in treatment $I$, the average payoff is somewhat higher for male subjects (3.58) than for female subjects (3.40), and both gender-specific payoff distributions differ significantly from the uniform distribution ( $p<0.001$, two-sided one-sample KS tests). Hence, females are somewhat less prone to lying than men, but the difference is not statistically significant $(p=0.477$, two-sided MWU test). Based on own calculations, this is again very similar to the baseline treatment of Fischbacher and Föllmi-Heusi (2013), where the respective gender-specific values are 3.60 and 3.37 with $p=0.133$.

The (slight) tendency of females to lie less than males is, however, amplified in treatment $G$, where we observe groups that are either "female" (only females), "male" (only males), or "mixed" (one female, one male). As illustrated in Fig. 1(b), compared to treatment $I$, the average payoff of female groups decreases (to 2.74), while the average payoff of male and mixed groups increases (to 4.00 and 3.71 , respectively). Payoffs of female groups are significantly lower than payoffs of male groups or mixed groups (pair-wise two-sided MWU tests with $p=0.045$ and $p=0.059$, respectively). The payoffs of male groups and mixed groups are not significantly different from each other (two-sided MWU test, $p=0.497$ ). A Jonckheere-Terpstra test indicates that the extent of lying is lowest for female groups followed by female individuals, male individuals, and male groups ( $p=0.026$, two-sided). In fact, while the payoff distributions of both male groups and mixed groups differ significantly from the uniform distribution, which

\footnotetext{
3 As participants still had to read the instructions in the booth, they did not need to worry that the time they spent there might be indicative of lying.

4 Chytilova and Korbel (2014) conduct an artefactual field experiment on lying with children and adolescents at a high school, where participants were paid in sweets. Their three-person groups obtain a somewhat higher payoff than individuals ( 3.28 and 2.93 , respectively).
} 
Table 1

Summary of payoffs.

\begin{tabular}{|c|c|c|c|c|c|c|c|c|}
\hline Treatment & $n$ & $\bar{\pi}$ & $\pi_{i}=0$ & $\pi_{i}=1$ & $\pi_{i}=2$ & $\pi_{i}=3$ & $\pi_{i}=4$ & $\pi_{i}=5$ \\
\hline I (all individuals) & 108 & 3.48 & $0.08^{--}$ & $0.06^{---}$ & $0.09^{--}$ & 0.19 & $0.28^{+++}$ & $0.31^{+++}$ \\
\hline$I$ (females only) & 58 & 3.40 & $0.05^{--}$ & 0.10 & 0.09 & 0.22 & 0.22 & $0.31^{+++}$ \\
\hline$I$ (males only) & 50 & 3.58 & 0.10 & $0.00^{---}$ & 0.10 & 0.14 & $0.34^{++}$ & $0.32^{+++}$ \\
\hline$G$ (all groups) & 60 & 3.47 & $0.05^{--}$ & 0.10 & 0.12 & 0.17 & 0.20 & $0.37^{+++}$ \\
\hline$G$ (female groups only) & 19 & 2.74 & 0.16 & 0.11 & 0.21 & 0.11 & 0.21 & 0.21 \\
\hline$G$ (male groups only) & 13 & 4.00 & 0.00 & 0.08 & 0.08 & 0.15 & 0.15 & $0.54^{+++}$ \\
\hline$G$ (mixed groups only) & 28 & 3.71 & $0.00^{---}$ & 0.11 & 0.07 & 0.21 & 0.21 & $0.39^{+++}$ \\
\hline
\end{tabular}

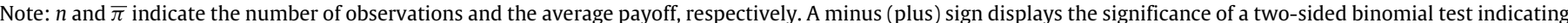
that the observed relative frequency is smaller (larger) than $\frac{1}{6}:^{-}\left({ }^{+}\right)=10 \%$-level, ${ }^{--}\left({ }^{++}\right)=5 \%$-level, ${ }^{---}\left({ }^{+++}\right)=1 \%$-level.

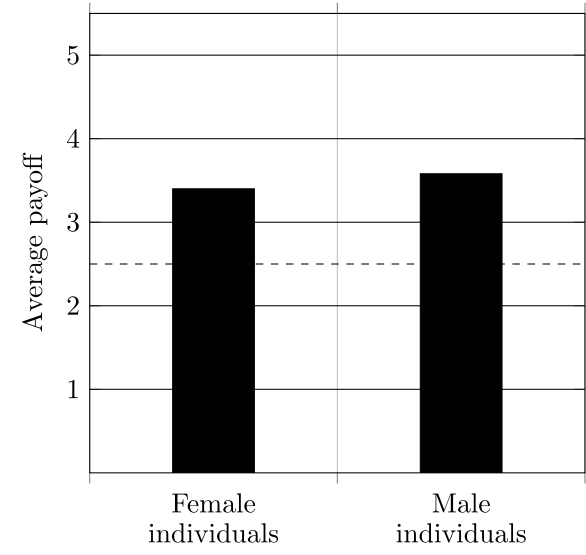

(a) Treatment $I$.

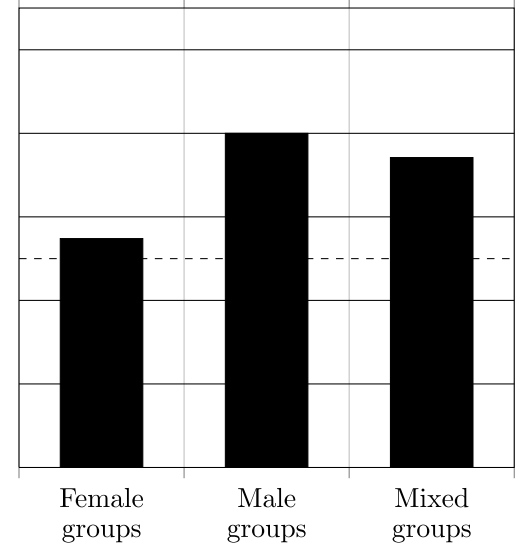

(b) Treatment $G$

Fig. 1. Average payoffs. The dotted line indicates a payoff of 2.50 , which would obtain on average under truthful reporting.

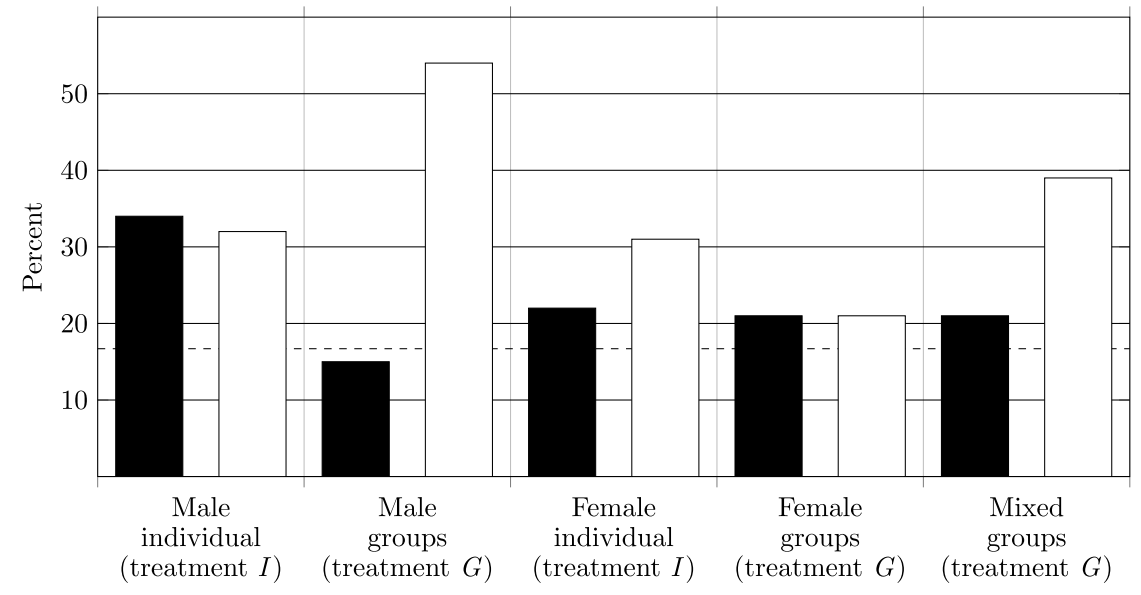

Fig. 2. Frequencies of 4's (black color) and 5's (white color) by gender and treatment.

would obtain under truthful reporting (two-sided one-sample KS tests, each with $p=0.001$ ), this is not the case for female groups ( $p=0.311$ ). That is, in contrast to individuals (either female or male), male groups, or mixed groups, one cannot reject that there is no lying in female groups.

There are also interesting gender differences with respect to the extent of lying, which we study by looking at the relative frequencies of 4's and 5's. ${ }^{5}$ First, we compare the behavior of male individuals and male groups, where similar fractions report either 4

\footnotetext{
5 In principle, subjects might also lie to their own disadvantage. However, at an aggregate level, for $\pi_{i} \leq 3$ none of the frequencies reported in Table 1 are significantly above the truth-telling benchmark $1 / 6$.
}

or 5 ( 0.66 and 0.69 , respectively). However, as illustrated by Fig. 2 , the fractions of male individuals who report 4 respectively 5 are almost identical. In contrast, male groups more often report 5 (in 54\% of cases) than 4 (in 15\% of cases), where this difference is significant at the $10 \%$-level of a one-sided binomial test that presumes that 4 and 5 occur with equal probability $(p=0.0898)$. Second, from comparing female individuals and female groups a different picture emerges. From Fig. 2, if anything, female groups are less likely to report 5's than female individuals (and in treatment $I(G)$ one cannot reject that 4's and 5's are reported by equal fractions of female individuals (female groups)). Finally, mixed groups seem to be more similar to male groups than to female groups, as there are more 5's than 4's in mixed groups (where the $p$-value of a respective one-sided binomial test is, however, only 0.1662 ). 


\section{Discussion}

Many important economic, social, or political decisions are taken by groups rather than individuals. We investigate how gender affects unethical behavior in the form of lying. In line with the previous literature, we find no clear evidence for gender differences under individual decision-making on lying. In contrast, in the case of group decision-making, more pronounced gender effects arise; resulting in more (less) aggregate unethical behavior in male (female) groups. Moreover, male groups seem to have a greater tendency towards exploiting the full gains from lying (i.e., securing the maximum payoff of 5) than female groups. Finally, mixed groups with equal shares of males and females behave similarly to male groups. Hence, from the viewpoint of organizational design, our results suggest that in contexts where unethical behavior might be an issue, designers might want to pay particular attention to decisions that are taken by purely male (or male-dominated) groups.

In future research, it would be interesting to study in more detail the forces underlying our results. In this respect, it is well known from other experimental settings that culture and cultural context might be important factors (see e.g., Roth et al., 1991; Cox et al., 1991; Herrmann et al., 2008). Cultural context might also be of relevance in our context of unethical behavior, and at least the following two channels might be at work: First, in the light of the discussion in Section 1, culture might influence how groups reach joint decisions as compared to individuals. For example, culture might affect how much group members are concerned with their "image" relative to the potential gains from acting dishonestly. Second, also gender differences in behavior might be driven by cultural context. Again, such effects have already been documented in other settings (see e.g., Finucane et al., 2000; Gneezy et al., 2009), and they might also be relevant in the context of unethical behavior.

\section{Acknowledgments}

Financial support by the Fritz Thyssen Foundation (Grant 10.13.2.097) is gratefully acknowledged. Moreover, we thank an anonymous referee, as well as Uta Czyrnick-Leber and Thomas Tröger for their helpful comments and suggestions. We also thank various research assistants, Ph.D. students, and post-docs for helping to run the experiment.

\section{Appendix. Experimental instructions}

\section{Supplementary Material: Instructions and Answer Sheet}

As Supplementary Material, we provide translated versions of the instructions (Appendix A.1) and the answer sheet (Appendix A.2) provided inside the booths for treatment $I$. In square brackets, we state the respective adjustments in the text for the two treatments I and G. The original versions (in German) are available upon request.

To avoid waiting lines at the cashier desks, we had two of them marked with green and red signs, respectively. We used otherwise identical, randomly allocated answer sheets and receipt forms in these colors (in equal proportions) and asked subjects to go to the corresponding color-coded cashier desk after they had completed the experiment.

\section{A.1. Instructions}

Please read the entire instructions first before you roll the die!

For participating in this experiment [G: each of] you will receive a payoff.
For organizational reasons, the color of your documents determines at which cashier desk you will receive your payoff: If your documents are in red, [G: both of] you may go to the red cashier desk after finishing the experiment. If they are in green, then [ $G$ : both of] you may go to the green cashier desk after finishing the experiment.

The payoff that [G: each of] you will receive will be determined by rolling a die:

\begin{tabular}{l|llllll} 
Outcome of die roll & 1 & 2 & 3 & 4 & 5 & 6 \\
\hline Payoff in Euros & 1 & 2 & 3 & 4 & 5 & 0
\end{tabular}

[ $I$ : Please roll the die in front of you once.] [G: Please agree upon who of you will roll the die in front of you once.] After that, please circle the outcome of the die roll and the related payoff on the answer sheet. You are free to roll the die more than once, but only the first roll is relevant for your payoff.

In a next step, we ask [ $G$ : each of] you to fill out and sign your receipt form (name and payoff) in line with your entry on the answer sheet.

[G: Together] please submit all documents ([I: questionnaire, answer sheet, receipt] [ $G$ : both questionnaires, answer sheet, receipts]) at the respective cashier desk. [G: Each of] you will receive [ $I$ : your] [G: his/her] payoff there.

If you have any questions, please contact a member of the support team. If not, please roll the die now.

Thank you for your participation!

\section{A.2. Answer sheet}

Please circle the combination of the outcome of the die roll and the corresponding payoff:

\begin{tabular}{l|llllll} 
Outcome of die roll & 1 & 2 & 3 & 4 & 5 & 6 \\
\hline Payoff in Euros & 1 & 2 & 3 & 4 & 5 & 0
\end{tabular}

\section{References}

Abeler, J., Becker, A., Falk, A., 2014. Representative evidence on lying costs. J. Public Econ. 113, 96-104.

Bornstein, G., Kugler, T., Ziegelmeyer, A., 2004. Individual and group decisions in the centipede game: are groups more "rational" players? J. Exp. Soc. Psychol. 40 (5), 599-605.

Charness, G., Dufwenberg, M., 2006. Promises and partnership. Econometrica 74 (6), 1579-1601.

Charness, G., Sutter, M., 2012. Groups make better self-interested decisions. J. Econ. Perspect. 26 (3), 157-176.

Childs, J., 2012. Gender differences in lying. Econom. Lett. 114 (2), 147-149.

Chytilova, J., Korbel, V., 2014. Individual and group cheating behavior: a field experiment with adolescents, IES Working Paper 06/2014, Charles University Prague.

Conrads, J., Irlenbusch, B., Rilke, R.M., Schielke, A., Walkowitz, G., 2014. Honesty in tournaments. Econom. Lett. 123 (1), 90-93.

Conrads, J., Irlenbusch, B., Rilke, R.M., Walkowitz, G., 2013. Lying and team incentives. J. Econ. Psychol. 34, 1-7.

Cox, T.H., Lobel, S.A., McLeod, P.L., 1991. Effects of ethnic group cultural differences on cooperative and competitive behavior on a group task. Acad. Manag. J. 34 (4), 827-847.

Croson, R., Gneezy, U., 2009. Gender differences in preferences. J. Econom. Lit. 47 (2), 448-474.

Dreber, A., Johannesson, M., 2008. Gender differences in deception. Econom. Lett. 99 (1), 197-199.

Dufwenberg, M., Muren, A., 2006. Gender composition in teams. J. Econ. Behav. Organ. 61 (1), 50-54.

Eckel, C.C., Grossman, P.J., 2008. Differences in the economic decisions of men and women: experimental evidence. In: Plott, C.R., Smith, V.L. (Eds.), Handbook of Experimental Economics Results, vol. 1. Elsevier.

Erat, S., Gneezy, U., 2012. White lies. Manage. Sci. 58 (4), 723-733.

Falk, A., Szech, N., 2013. Morals and markets. Science 340 (6133), 707-711.

Finucane, M.L., Slovic, P., Mertz, C.K., Flynn, J., Satterfield, T.A., 2000. Gender, race, and perceived risk: the 'white male' effect. Health Risk Soc. 2 (2), 159-172.

Fischbacher, U., Föllmi-Heusi, F., 2013. Lies in disguise: an experimental study on cheating. J. Eur. Econ. Assoc. 11 (3), 525-547. 
Gibson, R., Tanner, C., Wagner, A.F., 2013. Preferences for truthfulness: heterogeneity among and within individuals. Amer. Econ. Rev. 103 (1), 532-548.

Gneezy, U., 2005. Deception: the role of consequences. Amer. Econ. Rev. 95 (1) 384-394.

Gneezy, U., Leonard, K.L., List, J.A., 2009. Gender differences in competition: evidence from a matrilineal and a patriarchal society. Econometrica 77 (5), 1637-1664.

Herrmann, B., Thöni, C., Gächter, S., 2008. Antisocial punishment across societies. Science 319 (5868), 1362-1367.

Houser, D., Vetter, S., Winter, J., 2012. Fairness and cheating. Eur. Econ. Rev. 56 (8), 1645-1655.
Kugler, T., Kausel, E.E., Kocher, M.G., 2012. Are groups more rational than individuals? A review of interactive decision making in groups. Wiley Interdiscip. Rev. Cogn. Sci. 3 (4), 471-482.

Maciejovsky, B., Sutter, M., Budescu, D.V., Bernau, P., 2013. Teams make you smarter: how exposure to teams improves individual decisions in probability and reasoning tasks. Manage. Sci. 59 (6), 1255-1270.

Pruckner, G.J., Sausgruber, R., 2013. Honesty on the streets: a field study on newspaper purchasing. J. Eur. Econ. Assoc. 11 (3), 661-679.

Roth, A.E., Prasnikar, V., Okuno-Fujiwara, M., Zamir, S., 1991. Bargaining and market behavior in Jerusalem, Ljubljana, Pittsburgh, and Tokyo: an experimental study. Amer. Econ. Rev. 81 (5), 1068-1095. 